\title{
Impact of clinical parameters and systemic inflammatory status on epidermal growth factor receptor-mutant non-small cell lung cancer patients readministration with epidermal growth factor receptor tyrosine kinase inhibitors
}

Yu-Mu Chen ${ }^{1 \dagger}$, Chien-Hao Lai ${ }^{1 \dagger}$, Kun-Ming Rau ${ }^{2}$, Cheng-Hua Huang ${ }^{2}$, Huang-Chih Chang ${ }^{1}$, Tung-Ying Chao ${ }^{1}$, Chia-Cheng Tseng', Wen-Feng Fang 1,3, Yu-Hsiu Chung1', Yi-Hsi Wang1', Mao-Chang Su', Kuo-Tung Huang1, Shih-Feng Liu', Hung-Chen Chen ${ }^{1}$, Ya-Chun Chang ${ }^{1}$, Yu-Ping Chang ${ }^{1}$, Chin-Chou Wang ${ }^{1}$ and Meng-Chih Lin ${ }^{1 *}$

\begin{abstract}
Background: Epidermal growth factor receptor (EGFR)-tyrosine kinase inhibitor (TKI) readministration to lung cancer patients is common owing to the few options available. Impact of clinical factors on prognosis of EGFR-mutant non-small cell lung cancer (NSCLC) patients receiving EGFR-TKI readministration after first-line EGFR-TKI failure and a period of TKI holiday remains unclear. Through this retrospective study, we aimed to understand the impact of clinical factors in such patients.
\end{abstract}

Methods: Of 1386 cases diagnosed between December 2010 and December 2013, 80 EGFR-mutant NSCLC patients who were readministered TKls after failure of first-line TKls and intercalated with at least one cycle of cytotoxic agent were included. We evaluated clinical factors that may influence prognosis of TKI readministration as well as systemic inflammatory status in terms of neutrophil-to-lymphocyte ratio (NLR) and lymphocyte-to-monocyte ratio (LMR). Baseline NLR and LMR were estimated at the beginning of TKI readministration and trends of NLR and LMR were change amount from patients receiving first-Line TKls to TKls readministration.

Results: Median survival time since TKI readministration was 7.0 months. In the univariable analysis, progression free survival (PFS) of first-line TKIs, baseline NLR and LMR, and trend of LMR were prognostic factors in patients receiving TKls readministration. In the multivariate analysis, only PFS of first-line TKIs $(p<0.001)$, baseline NLR $(p=0.037)$, and trend of LMR $(p=0.004)$ were prognostic factors.

Conclusion: Longer PFS of first-line TKIs, low baseline NLR, and high trend of LMR were good prognostic factors in EGFR-mutant NSCLC patients receiving TKI readministration.

Keywords: Neutrophil-to-lymphocyte ratio, Lymphocyte-to-monocyte ratio, Readministration, Non-small cell lung cancer, Epidermal growth factor receptor, Tyrosine kinase inhibitor

\footnotetext{
* Correspondence: linmengchih@hotmail.com

${ }^{\dagger}$ Equal contributors

${ }^{1}$ Division of Pulmonary and Critical Care Medicine, Department of Internal

Medicine, Chang Gung Memorial Hospital-Kaohsiung Medical Center, Chang

Gung University College of Medicine, No. 123, Ta-Pei Road, Niao-Sung

District, Kaohsiung City, Taiwan

Full list of author information is available at the end of the article
} 


\section{Background}

Lung cancer is the leading cause of cancer-related deaths in Taiwan and worldwide [1, 2]. Although epidermal growth factor receptor (EGFR)-tyrosine kinase inhibitors (TKIs) are administered as standard first-line regimen for advanced EGFR-mutant non-small cell lung cancer (NSCLC) [3-5], the salvage treatment for cases with acquired resistance to EGFR-TKIs remains unclear. Owing to several barriers including difficulty of tumor re-biopsy, absence of EGFR T790m mutation or programmed deathligand 1 expression, and high expenses, some patients do not have an opportunity to receive novel agents such as $3^{\text {rd }}$ generation TKI [6] or immunotherapies [7, 8].

In patients with acquired resistance to EGFR-TKIs, readministration of first or second generation EGFR-TKIs has been proved to effectively increase patients' survival time [9-11]. In non-selective patients, EGFR-TKI readministration has only modest efficacy with a progression free survival (PFS) of 2-4 months [10, 12]. However, in optimal selected patients, patients could have a PFS of more than 6 months [10]. Although several good prognostic factors for patients receiving TKI readministration have been reported, such as EGFR-TKI free holidays, better Eastern Cooperative Oncology Group performance status, and benefit from prior EGFR-TKI therapy [10-12], little is known about the correlation between systemic inflammatory markers and TKI readministration efficacies. In previous studies, several systemic inflammatory markers were found to be prognostic factors in lung cancer patients. NSCLC patients with higher blood neutrophil-tolymphocyte ratio (NLR) had poor prognosis when treated with a combination of bevacizumab and cytotoxic agents [13]; those with higher lymphocyte-to-monocyte ratio (LMR) had better prognosis in EGFR-mutant NSCLC patients receiving first-line EGFR-TKIs [14].

Based on these aforementioned reasons we performed a retrospective study to understand the impact of clinical factors including NLR and LMR on EGFR-mutant NSCLC patients receiving TKI readministration. To decrease the impact of confounding factors, we only included EGFRmutant NSCLC patients receiving TKI readministration as third or later line therapies after failure of first-line EGFRTKIs and at least one cycle of intercalated chemotherapy.

\section{Methods}

\section{Patients and clinical characteristics}

We conducted a retrospective study between December 2010 and December 2013 at Kaohsiung Chang Gung Memorial Hospital in Taiwan. Patients were followed-up until November 2015. Adult patients aged $\geq 18$ years with histologically or cytologically confirmed stage IIIB or IV NSCLC who had been treated with first line EGFR-TKIs and received TKIs readministration were included. Patients who had received a second TKI without intercalating with at least one cycle of cytotoxic chemotherapies were excluded.

Baseline assessments including clinical parameters, hematological variables, biochemistry, chest radiography, and chest computed tomography were performed within 4 weeks of initiation of TKI readministration.

Clinical parameters included length of TKI holiday and PFS of study patients receiving first line EGFR-TKIs. Data regarding hematological parameters were collected within 4 weeks of the initiation of first-line TKI therapy and also TKI readministration including neutrophil, lymphocyte, and monocyte counts. NLR was obtained by dividing the neutrophil count by the lymphocyte count, and LMR was obtained by dividing the lymphocyte count by the monocyte count. Baseline NLR and LMR were estimated at the beginning of TKI readministration and trends of NLR and LMR were obtained by dividing the data estimated at the beginning of TKI readministration with the data estimated at the beginning of first-line TKIs.

This study was approved by the Institutional Review Board of Kaohsiung Chang Gung Memorial Hospital. The need for informed consent was waived.

\section{EGFR mutation testing}

Tumor specimens were obtained by bronchoscopy CTguided biopsy, pleural effusion cytology, or surgical procedures. The EGFR mutational analyses was performed using SCORPIONS and ARMS polymerase chain reaction using fragments amplified from genomic DNA extracted from paraffin-embedded tissues (QIAGEN EGFR RGQ PCR KIT). Exon 19 deletion and L858R mutations were defined as common mutations. Other mutations or compound mutations were defined as uncommon mutations.

\section{Evaluation of response to EGFR-TKI readministration}

Patients underwent routine chest radiography every 2-4 weeks and chest computed tomography every 23 months to evaluate tumor responses. PFS was defined as the time between the first day of EGFR-TKI administration and disease progression, death before documented progression, or the last visit during the follow-up period. Disease progression was determined by the clinician according to the Response Evaluation Criteria in Solid Tumors criteria 1.1 [15]. The endpoint was overall survival (OS), which was defined as the first day of EGFR-TKI readministration until death, or the last visit during the follow-up period.

\section{Statistical analyses}

Statistical analyses were performed using MedCalc (version 14.10.2). Receiver operating characteristic (ROC) curves with binary variable of OS longer or shorter than 7.0 months since readministration and Youden's index were used to determine the best cut-off value for baseline 
values of and trends of NLR LMR as a prognostic factors. OS analyses were performed using the Kaplan-Meier method and the log-rank test. Cox proportional hazards regression test were used to evaluate independent factors. $P$ value $<0.05$ was considered significant in statistical tests.

\section{Results}

\section{Patient characteristics}

Between December 2010 and December 20131386 lung cancer cases were diagnosed. Of these, 269 patients had a positive EGFR mutation status and were treated with firstline EGFR-TKIs, and 80 patients were readministered TKIs with at least one cycle intercalated cytotoxic agent (Fig. 1). Lines and regimens of Intercalated chemotherapies were

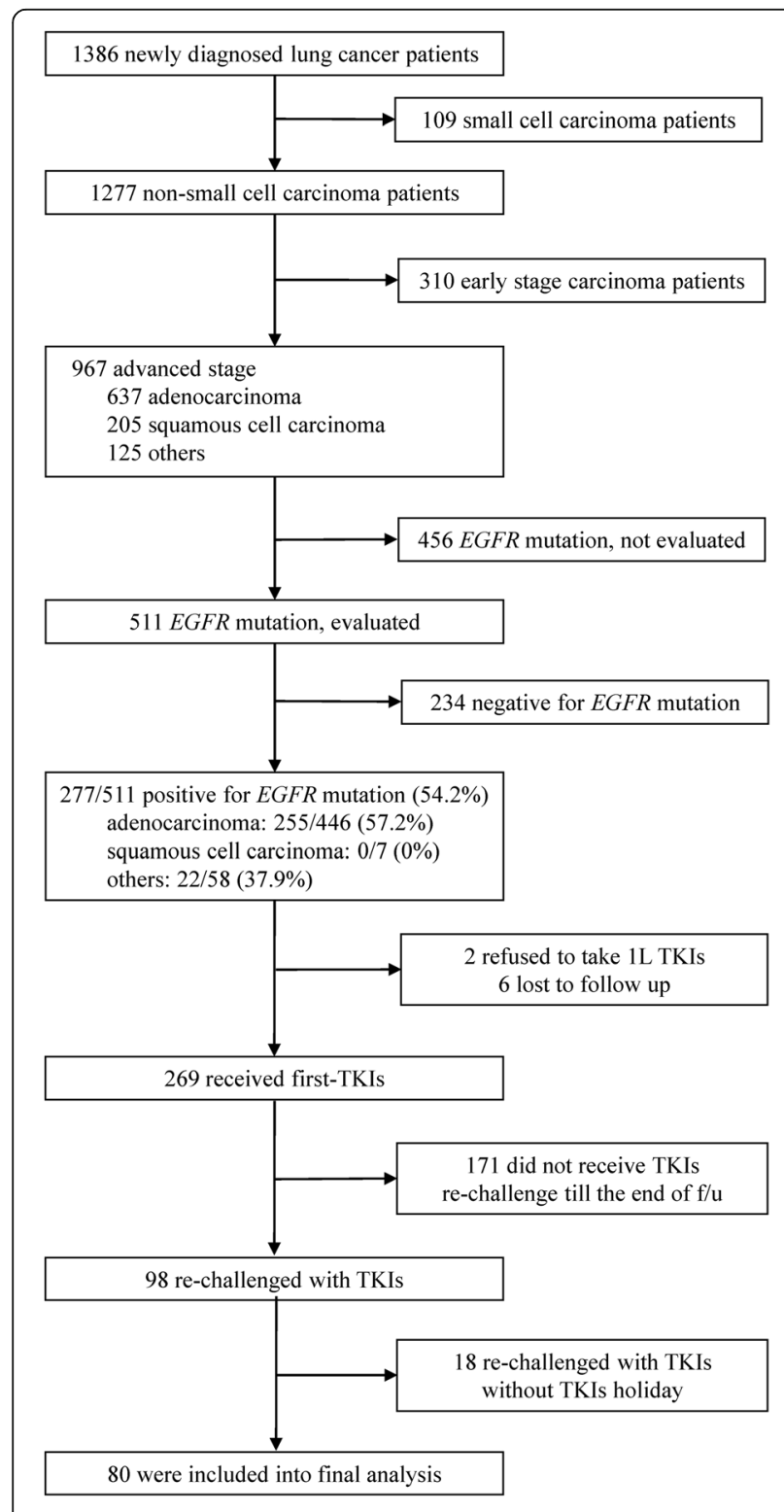

Fig. 1 Inclusion, screening, and assignment of patients into groups shown in Additional file 1: Table S1. The median follow-up time since readministration was 7.0 months the longest follow-up duration was 20.4 months. At the end of followup $78.8 \%$ (63/80) patients showed disease progression under TKI readministration and $36.3 \%$ (29/80) patients were alive. Baseline values and trends of hematological parameters were available for 78 and 77 patients, respectively. To evaluate baseline values and trends of NLR and LMR, using ROC curve analysis, we determined that the best cut-off values were $5.2,1.1,2.5$, and 0.5 , respectively.

\section{Impact of clinical factors on overall survival of TKI readministration}

Clinical factors found to be significant in the univariable analysis for poor OS since TKI readministration included shorter PFS of first-line TKI $(p=0.020)$ (Fig. 2) high baseline NLR $(p<0.001)$ (Fig. 3a), low baseline LMR $(p=0.006 \mathrm{~B})$, and low trend of LMR $(p=0.037)$ (Fig. 4) (Table 1).

Length of TKI holiday changes in the TKI regimen, and first or second generation TKIs when TKI readministration, and trend of NLR did not significantly influence OS. In the multivariable analysis, independent prognostic factors for shorter OS were shorter first-line TKI PFS $(p<0.001)$, high baseline NLR $(p=0.037)$, and low trend of LMR $(p=0.004)$ (Table 1).

\section{Discussion}

Our retrospective observational study found that baseline NLR and trend of LMR as well as PFS of first-line EGFR-TKI treatment were prognostic factors in patients receiving TKI readministration. NLR was previously found to have a prognostic effect in different types of cancer like ovarian cancer, breast cancer, pancreatic

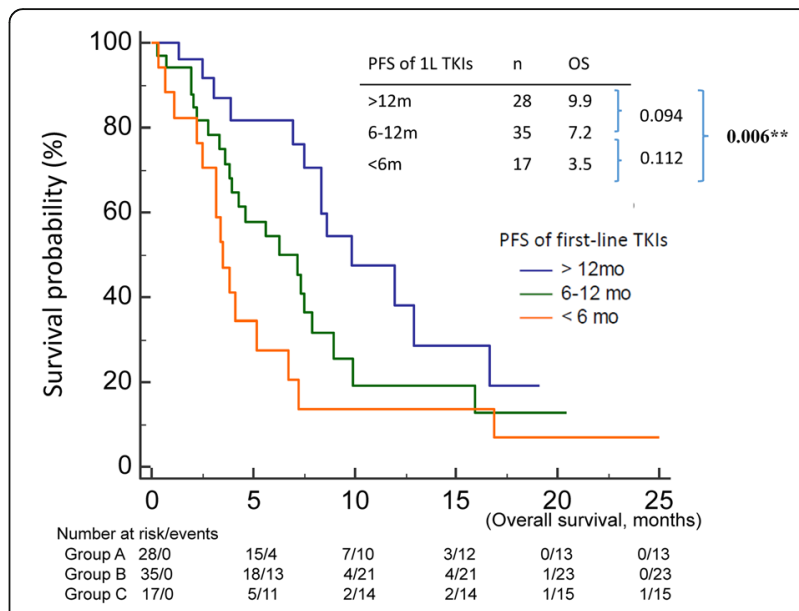

Fig. 2 Overall survival since the readministration of tyrosine kinase inhibitors of patients with short ( $<6$ months), intermediate (6-12 months), and long (>12 months) progression free survival of first-line tyrosine kinase inhibitors 


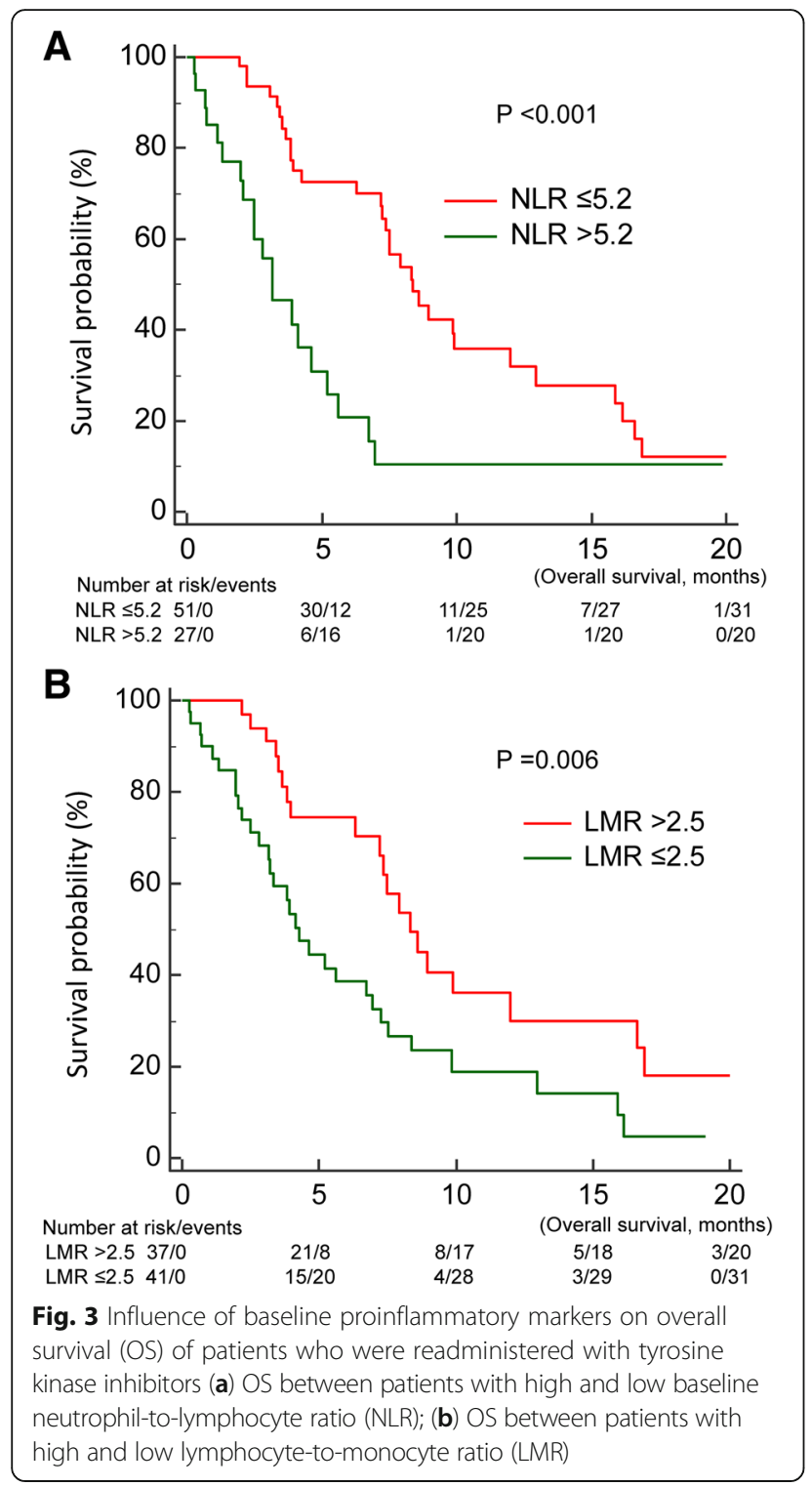

cancer, and colorectal cancer, as well as in advanced NSCLC patients treated with first-line platinum-based chemotherapy [16-21]. LMR was found to be a prognostic factor in small cell lung cancer [22], in early-stage NSCLC patients post operation [23], in advanced lung cancer treated with cytotoxic chemotherapies [24], and in EGFR-mutant lung cancer patients treated with firstline EGFR-TKIs [14]. Several possible mechanisms may explain the prognostic effect of these pro-inflammatory markers. First, neutrophils release several pro-angiogenic factors and promote angiogenesis, which is essential for tumor progression. Second, lymphocytes play a pivotal role in tumor cell eradication [25], and tumor-associated macrophages promote tumor progression through remodeling of the tumor extracellular matrix [26, 27]. Based on the above pathophysiology, patients with high NLR and low

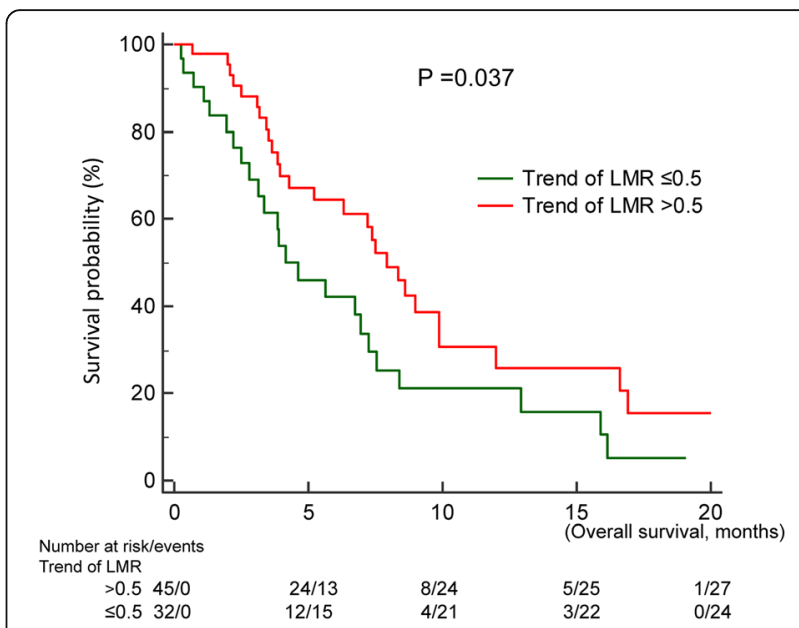

Fig. 4 Influence of trends of lymphocyte-to-monocyte ratio (LMR) on overall survival (OS) of patients who were readministered with tyrosine kinase inhibitors OS between patients with high and low trend of LMR

LMR tend to have tumor progression and fewer T cells available for cancer cell eradication.

Previous studies have reported conflicting results regarding the influence of PFS of previous EGFR-TKI on the efficacy of TKI readministration. In one study that included all patients without TKI holidays longer PFS of previous TKI treatment paradoxically shortened the PFS of TKI readministration [11]. Another study in which $52 \%$ of patients with a TKI holiday before TKI rechallenge revealed that PFS of previous TKI treatment was not related with the efficacy of TKI readministration [12]. By excluding patients without having TKIs holidays, our study revealed that patients with a longer PFS of previous TKI treatment have a longer OS of TKI readministration. In the first study, the authors speculated that in patients who received previous therapy for less than 12 months, the tumor may not yet have acquired the $790 \mathrm{M}$ mutation. However, this concept was not supported by subsequent studies [28]. We speculated that when the disease progresses after the first TKI therapy, tumors have a dominant part of TKI-resistant clones and a minor part of TKI-sensitive clones.

After the TKI holidays and owing to intercalation with cytotoxic chemotherapies tumor redistribution occurred, which lead to TKI-sensitive clones increasing, and TKIresistant clones decreasing. This redistribution was due to higher sensitivity to cytotoxic chemotherapies in TKIresistant clones than that in TKI-sensitive clones. After tumor redistribution by the intercalated chemotherapies, tumor characteristics were more similar to those of TKI-naïve tumors than to TKI-resistant tumors.

This can explain at least partly, why PFS of previous TKIs has opposite influences in patients with or without 
Table 1 Clinical factors and systemic inflammatory status of patients receiving EGFR-TKI readministration

\begin{tabular}{|c|c|c|c|c|c|c|}
\hline \multirow[b]{2}{*}{ Characteristics } & \multicolumn{3}{|c|}{ Univariable analyses } & \multicolumn{3}{|c|}{ Multivariable analyses } \\
\hline & N (\%) & OS & $\mathrm{p}$ & Hazard ratio & $95 \% \mathrm{Cl}$ & $P$ value \\
\hline Length of EGFR-TKI holiday & & & 0.235 & & & \\
\hline$<3$ & $16(20.0)$ & 3.8 & & & & \\
\hline $3-6$ & $25(31.3)$ & 6.7 & & & & \\
\hline$>6$ & $39(48.8)$ & 8.4 & & & & \\
\hline PFS of first-line EGFR-TKI & & & 0.020 & & & $<0.001$ \\
\hline$<6$ & $17(35.0)$ & 3.5 & & 4.970 & $2.170-11.382$ & \\
\hline $6-12$ & $35(43.8)$ & 7.2 & & 1.818 & $0.899-3.678$ & \\
\hline$>12$ & $28(21.3)$ & 9.9 & & 1 & & \\
\hline Changes in the EGFR-TKI regimen & & & 0.474 & & & \\
\hline Yes & $75(93.8)$ & 7.2 & & & & \\
\hline No & $5(6.2)$ & 8.4 & & & & \\
\hline Type of EGFR-TKI readministrated & & & 0.934 & & & \\
\hline $1^{\text {st }}$ generation & $71(88.8)$ & 7.0 & & & & \\
\hline $2^{\text {nd }}$ generation & $9(11.2)$ & 7.4 & & & & \\
\hline Baseline NLR & & & $<0.001$ & & & 0.037 \\
\hline$>5.2$ & $27(34.6)$ & 3.2 & & 2.352 & $1.052-5.256$ & \\
\hline$\leq 5.2$ & $51(65.4)$ & 8.4 & & 1 & & \\
\hline Trend of NLR & & & 0.129 & & & \\
\hline$>110 \%$ & $44(57.1)$ & 4.3 & & & & \\
\hline$\leq 110 \%$ & $33(42.9)$ & 8.4 & & & & \\
\hline Baseline LMR & & & 0.006 & & & 0.632 \\
\hline$>2.5$ & $37(46.8)$ & 8.3 & & 1 & & \\
\hline$\leq 2.5$ & $41(53.2)$ & 4.2 & & 1.197 & $0.574-2.497$ & \\
\hline Trend of LMR & & & 0.037 & & & 0.004 \\
\hline$>50 \%$ & $45(58.4)$ & 7.9 & & 1 & & \\
\hline$\leq 50 \%$ & $32(41.6)$ & 4.1 & & 2.651 & $1.374-5.118$ & \\
\hline
\end{tabular}

Abbreviations: Cl confidential interval, EGFR epidermal growth factor receptor, LMR lymphocyte to monocyte ratio, NLR neutrophil to lymphocyte ratio, OS overall survival, PFS progression-free survival, TKI tyrosine kinase inhibitor

TKI holidays. However, this concept should be proved with further studies.

Though several studies have reported on how clinical factors affect the efficacies of TKI readministration [10-12] patient heterogeneity is a confounding factor that cannot be neglected. One study included more than $70 \%$ of patients without TKI holidays, whereas two other studies included 32 and $50 \%$ patients with wild type EGFR mutation, respectively. We only included EGFR-mutant NSCLC patients receiving firstline EGFR-TKIs and at least one intercalated chemotherapy agent to decrease these confounding factors. To the best of our knowledge, this is the first study demonstrating that baseline NLR and trend of LMR are prognostic factors in patients receiving EGFR-TKI readministration. As a study aimed at patients receiving third and later line therapies, the number of patients is not small.
Our study had several limitations. First, data regarding the amount and pattern of inflammatory cell infiltration as well as the amount of tumor programmed death-ligand 1 expression in tumors were not available, which could have provided us further information about the immune condition in the tumor microenvironment [29]. Further studies are required to determine whether immunotherapy or anti-angiogenesis agents could prolong survival in those who were speculated to have poor prognosis to TKI readministration. Finally, our study was a retrospective study a prospective trial is needed to validate these results.

\section{Conclusion}

Longer PFS of first-line TKIs, low baseline NLR, and high trend of LMR were good prognostic factors in EGFRmutant NSCLC patients receiving TKI readministration. 


\section{Additional file}

Additional file 1: Lines and regimens of Intercalated chemotherapies. (DOCX $12 \mathrm{~kb}$ )

\section{Abbreviations}

EGFR: Epidermal growth factor receptor; LMR: Lymphocyte-to-monocyte ratio; NLR: Neutrophil-to-lymphocyte ratio; NSCLC: Non-small cell lung cancer; OS: Overall survival; PFS: Progression free survival; ROC: Receiver operating characteristic; TKI: Tyrosine kinase inhibitor

\section{Acknowledgements}

We thank Tsui-Ping Tang and I-Chun Lin for the valuable assistance with data collection.

\section{Funding}

The authors have no support or funding to report.

\section{Availability of data and materials}

The raw of the data would not be shared at this moment because there are several papers under preparation based on this raw data.

\section{Authors' contributions}

Conceived and designed the experiments: YMC CHL HC Chang TYC CCT WFF SFL HC Chen YCC YPC MCL. Analyzed the data: YMC CCW YHC MCL. Contributed materials/analysis tools: YMC KMR CHH YHW MCL MCS KTH. Wrote the paper: YMC MCL. All authors read and approved the final manuscript.

\section{Competing interests}

The authors declare that they have no competing interests.

\section{Consent for publication}

Not applicable.

\section{Ethics approval and consent to participate}

This study was approved by the Institutional Review Board of Kaohsiung Chang Gung Memorial Hospital. IRB number: 102-4571b. The need for informed consent was waived. (The data were analyzed retrospectively, and all identifying patient data were removed prior to analysis.)

\section{Author details}

'Division of Pulmonary and Critical Care Medicine, Department of Internal Medicine, Chang Gung Memorial Hospital-Kaohsiung Medical Center, Chang Gung University College of Medicine, No. 123, Ta-Pei Road, Niao-Sung District, Kaohsiung City, Taiwan. ${ }^{2}$ Division of Hematology-Oncology, Department of Internal Medicine, Kaohsiung Chang Gung Memorial Hospital and Chang Gung University College of Medicine, Kaohsiung, Taiwan. ${ }^{3}$ Department of Respiratory Care, Chang Gung University of Science and Technology, Chiayi Campus, Chiayi, Taiwan.

\section{Received: 8 February 2016 Accepted: 31 October 2016}

Published online: 08 November 2016

\section{References}

1. Henley SJ, Richards TB, Underwood JM, Eheman CR, Plescia M, McAfee TA Centers for Disease C, Prevention. Lung cancer incidence trends among men and women-United States, 2005-2009. MMWR Morb Mortal Wkly Rep. 2014;63(1):1-5

2. Wang BY, Huang JY, Cheng CY, Lin CH, Ko J, Liaw YP. Lung cancer and prognosis in taiwan: a population-based cancer registry. J Thorac Oncol. 2013:8(9):1128-35.

3. Zhou C, Wu YL, Chen G, Feng J, Liu XQ, Wang C, Zhang S, Wang J, Zhou S, Ren $S$, et al. Final overall survival results from a randomised, phase III study of erlotinib versus chemotherapy as first-line treatment of EGFR mutationpositive advanced non-small-cell lung cancer (OPTIMAL, CTONG-0802). Ann Oncol. 2015;26(9):1877-83.

4. Fukuoka $\mathrm{M}, \mathrm{Wu} \mathrm{YL}$, Thongprasert $\mathrm{S}$, Sunpaweravong $\mathrm{P}$, Leong SS, Sriuranpong V, Chao TY, Nakagawa K, Chu DT, Saijo N, et al. Biomarke analyses and final overall survival results from a phase III, randomized, openlabel, first-line study of gefitinib versus carboplatin/paclitaxel in clinically selected patients with advanced non-small-cell lung cancer in Asia (IPASS). J Clin Oncol. 2011:29(21):2866-74.

5. Wu YL, Zhou C, Hu CP, Feng J, Lu S, Huang Y, Li W, Hou M, Shi JH, Lee KY, et al. Afatinib versus cisplatin plus gemcitabine for first-line treatment of Asian patients with advanced non-small-cell lung cancer harbouring EGFR mutations (LUX-Lung 6): an open-label, randomised phase 3 trial. Lancet Oncol. 2014;15(2):213-22.

6. Janne PA, Yang JC, Kim DW, Planchard D, Ohe Y, Ramalingam SS, Ahn MJ, Kim SW, Su WC, Horn L, et al. AZD9291 in EGFR inhibitor-resistant nonsmall-cell lung cancer. N Engl J Med. 2015;372(18):1689-99.

7. Brahmer J, Reckamp KL, Baas P, Crino L, Eberhardt WE, Poddubskaya E, Antonia S, Pluzanski A, Vokes EE, Holgado E, et al. Nivolumab versus Docetaxel in Advanced Squamous-Cell Non-Small-Cell Lung Cancer. N Engl J Med. 2015;373(2):123-35.

8. Garon EB, Rizvi NA, Hui R, Leighl N, Balmanoukian AS, Eder JP, Patnaik A Aggarwal C, Gubens M, Horn L, et al. Pembrolizumab for the treatment of non-small-cell lung cancer. N Engl J Med. 2015;372(21):2018-28.

9. Hata A, Katakami N, Kaji R, Fujita S, Imai Y. Does T790M disappear? Successful gefitinib rechallenge after T790M disappearance in a patient with EGFR-mutant non-small-cell lung cancer. J Thorac Oncol. 2013;8(3):e27-9.

10. Zhao ZR, Li W, Long H. Readministration of EGFR tyrosine kinase inhibitor in non-small cell lung cancer patients after initial failure, what affects its efficacy? Sci Rep. 2014;4:5996.

11. Asami K, Kawahara M, Atagi S, Kawaguchi T, Okishio K. Duration of prior gefitinib treatment predicts survival potential in patients with lung adenocarcinoma receiving subsequent erlotinib. Lung Cancer. 2011;73(2): 211-6.

12. Hata A, Katakami N, Yoshioka H, Fujita S, Kunimasa K, Nanjo S, Otsuka K, Kaji $\mathrm{R}$, Tomii K, Iwasaku M, et al. Erlotinib after gefitinib failure in relapsed nonsmall cell lung cancer: clinical benefit with optimal patient selection. Lung Cancer. 2011;74(2):268-73.

13. Botta C, Barbieri V, Ciliberto D, Rossi A, Rocco D, Addeo R, Staropoli N, Pastina P, Marvaso G, Martellucci I, et al. Systemic inflammatory status at baseline predicts bevacizumab benefit in advanced non-small cell lung cancer patients. Cancer Biol Ther. 2013;14(6):469-75.

14. Chen YM, Lai CH, Chang HC, Chao TY, Tseng CC, Fang WF, Wang CC, Chung YH, Wang YH, Su MC, et al. Baseline and Trend of Lymphocyte-toMonocyte Ratio as Prognostic Factors in Epidermal Growth Factor Receptor Mutant Non-Small Cell Lung Cancer Patients Treated with First-Line Epidermal Growth Factor Receptor Tyrosine Kinase Inhibitors. PLoS One. 2015:10(8):e0136252.

15. Eisenhauer EA, Therasse $P$, Bogaerts J, Schwartz LH, Sargent D, Ford R, Dancey J, Arbuck S, Gwyther S, Mooney M, et al. New response evaluation criteria in solid tumours: revised RECIST guideline (version 1.1). Eur J Cancer 2009:45(2):228-47.

16. Williams KA, Labidi-Galy SI, Terry KL, Vitonis AF, Welch WR, Goodman A, Cramer DW. Prognostic significance and predictors of the neutrophil-tolymphocyte ratio in ovarian cancer. Gynecol Oncol. 2014;132(3):542-50.

17. Jia W, Wu J, Jia H, Yang Y, Zhang X, Chen K, Su F. The Peripheral Blood Neutrophil-To-Lymphocyte Ratio Is Superior to the Lymphocyte-ToMonocyte Ratio for Predicting the Long-Term Survival of Triple-Negative Breast Cancer Patients. PLoS One. 2015;10(11):e0143061.

18. Szkandera J, Stotz M, Eisner F, Absenger G, Stojakovic T, Samonigg H, Kornprat P, Schaberl-Moser R, Alzoughbi W, Ress AL, et al. External validation of the derived neutrophil to lymphocyte ratio as a prognostic marker on a large cohort of pancreatic cancer patients. PLoS One. 2013; 8(11):e78225.

19. Cheng $H$, Long $F$, Jaiswar $M$, Yang L, Wang C, Zhou Z. Prognostic role of the neutrophil-to-lymphocyte ratio in pancreatic cancer: a meta-analysis. Sci Rep. 2015;5:11026.

20. Malietzis G, Giacometti M, Askari A, Nachiappan S, Kennedy RH, Faiz OD, Aziz O, Jenkins JT. A preoperative neutrophil to lymphocyte ratio of 3 predicts disease-free survival after curative elective colorectal cancer surgery. Ann Surg. 2014;260(2):287-92.

21. Yao Y, Yuan D, Liu H, Gu X, Song Y. Pretreatment neutrophil to lymphocyte ratio is associated with response to therapy and prognosis of advanced non-small cell lung cancer patients treated with first-line platinum-based chemotherapy. Cancer Immunol Immunother. 2013:62(3):471-9.

22. Go SI, Kim RB, Song HN, Kang MH, Lee US, Choi HJ, Lee SJ, Cho YJ, Jeong YY, Kim HC, et al. Prognostic significance of the lymphocyte-to-monocyte ratio in patients with small cell lung cancer. Med Oncol. 2014;31(12):323. 
23. Hu P, Shen H, Wang G, Zhang P, Liu Q, Du J. Prognostic significance of systemic inflammation-based lymphocyte- monocyte ratio in patients with lung cancer: based on a large cohort study. PLoS One. 2014;9(9):e108062.

24. Song YJ, Wang LX, Hong YQ, Lu ZH, Tong Q, Fang XZ, Tan J. Lymphocyte to monocyte ratio is associated with response to first-line platinum-based chemotherapy and prognosis of early-stage non-small cell lung cancer patients. Tumour Biol. 2016;37(4):5285-93.

25. Aerts JG, Hegmans JP. Tumor-specific cytotoxic T cells are crucial for efficacy of immunomodulatory antibodies in patients with lung cancer. Cancer Res. 2013;73(8):2381-8.

26. Yang J, Liao D, Chen C, Liu Y, Chuang TH, Xiang R, Markowitz D, Reisfeld RA, Luo Y. Tumor-associated macrophages regulate murine breast cancer stem cells through a novel paracrine EGFR/Stat3/Sox-2 signaling pathway. Stem Cells. 2013;31(2):248-58.

27. Lin EY, Li JF, Gnatovskiy L, Deng Y, Zhu L, Grzesik DA, Oian H, Xue XN, Pollard JW. Macrophages regulate the angiogenic switch in a mouse model of breast cancer. Cancer Res. 2006;66(23):11238-46.

28. Ohashi K, Maruvka YE, Michor F, Pao W. Epidermal growth factor receptor tyrosine kinase inhibitor-resistant disease. J Clin Oncol. 2013;31(8):1070-80

29. Remark R, Becker C, Gomez JE, Damotte D, Dieu-Nosjean MC, SautesFridman C, Fridman WH, Powell CA, Altorki NK, Merad M, et al. The nonsmall cell lung cancer immune contexture. A major determinant of tumor characteristics and patient outcome. Am J Respir Crit Care Med. 2015;191(4): 377-90.

\section{Submit your next manuscript to BioMed Central} and we will help you at every step:

- We accept pre-submission inquiries

- Our selector tool helps you to find the most relevant journal

- We provide round the clock customer support

- Convenient online submission

- Thorough peer review

- Inclusion in PubMed and all major indexing services

- Maximum visibility for your research

Submit your manuscript at www.biomedcentral.com/submit

) Biomed Central 